\title{
Comparison of rectal volume definition techniques and their influence on rectal toxicity in patients with prostate cancer treated with 3D conformal radiotherapy: a dose-volume analysis

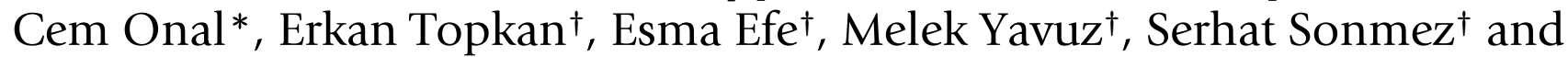 Aydin Yavuz ${ }^{\dagger}$
}

Address: Department of Radiation Oncology, Baskent University Medical Faculty, Adana, Turkey

Email: Cem Onal* - hcemonal@hotmail.com; Erkan Topkan - drerkantopkan@yahoo.com; Esma Efe - efeesma@gmail.com; Melek Yavuz - mayvuz@baskent-adn.edu.tr; Serhat Sonmez - serhatsnmzy@yahoo.com; Aydin Yavuz - ayavuz@baskent-adn.edu.tr

* Corresponding author †Equal contributors

Published: II May 2009

Radiation Oncology 2009, 4:14 doi:10.1186/1748-717X-4-14

This article is available from: http://www.ro-journal.com/content/4/I/I4

(c) 2009 Onal et al; licensee BioMed Central Ltd.

This is an Open Access article distributed under the terms of the Creative Commons Attribution License (http://creativecommons.org/licenses/by/2.0), which permits unrestricted use, distribution, and reproduction in any medium, provided the original work is properly cited.
Received: 17 February 2009

Accepted: II May 2009

\begin{abstract}
Background: To evaluate the impact of four different rectum contouring techniques and rectal toxicities in patients with treated with 3D conformal radiotherapy (3DCRT).

Methods: Clinical and dosimetric data were evaluated for 94 patients who received a total dose 3DCRT of $70 \mathrm{~Gy}$, and rectal doses were compared in four different rectal contouring techniques: the prostate-containing CT sections (method I); I cm above and below the planning target volume (PTV) (method 2); $110 \mathrm{~mm}$ starting from the anal verge (method 3); and from the anal verge to the sigmoid flexure (method 4). The percentage of rectal volume receiving RT doses (30-70 Gy) and minimum, mean rectal doses were assessed.
\end{abstract}

Results: Median age was 69 years. Percentage of rectal volume receiving high doses ( $\geq 70 \mathrm{~Gy}$ ) were higher with the techniques that contoured smaller rectal volumes. In methods 2 and 3, the percentage of rectal volume receiving $\geq 70 \mathrm{~Gy}$ was significantly higher in patients with than without rectal bleeding (method 2: $30.8 \%$ vs. $22.5 \%$, respectively ( $p=0.03$ ); method $3: 26.9 \%$ vs. $18.1 \%$, respectively $(p=0.006)$ ). Mean rectal dose was significant predictor of rectal bleeding only in method 3 (48.8 Gy in patients with bleeding vs. $44.4 \mathrm{~Gy}$ in patients without bleeding; $\mathrm{p}=0.02$ ).

Conclusion: Different techniques of rectal contouring significantly influence the calculation of radiation doses to the rectum and the prediction of rectal toxicity. Rectal volume receiving higher doses ( $\geq 70 \mathrm{~Gy}$ ) and mean rectal doses may significantly predict rectal bleeding for techniques contouring larger rectal volumes, as was in method 3.

\section{Background}

Prostate cancer is a radio-responsive tumor with a welldefined dose-response relationship $[1,2]$. Higher radiotherapy (RT) doses have been associated with better biochemical control rates and fewer distant relapses $[1,3]$.
Those findings support the suggestion that enhanced survival rates may be achievable with an improvement in local control. However, the use of higher RT doses is limited by an increased risk of complications in adjacent normal tissues. In this setting, more sophisticated techniques 
such as three dimensional conformal RT (3DCRT), intensity modulated RT (IMRT), and tomotherapy allow more precise treatment planning with better sparing of the normal tissues [4], which yields higher local control with significant reduction in both acute and late complications [5] Nevertheless, the use of higher RT doses beyond the conventional doses has been demonstrated to cause a moderate increase in the dose-limiting, late rectal toxicity, mainly manifested by rectal bleeding $[6,7]$.

The major predictor of rectal bleeding is the volume of the rectum included in the high dose region $[8,9]$, and the correlation between rectal bleeding rates and the irradiated rectal volume has been well established [9-12]. Furthermore dose-volume histograms (DVH) served as useful tools in demonstrating this significant relationship. Despite its extreme importance, no universally accepted method has been established for rectal contouring in RT planning for prostatic carcinomas. The length of rectum contoured has been defined in different ways by different authors. Examples of these definitions include: $1 \mathrm{~cm}$ above and below the planning target volume (PTV) $[13,14]$, the length of the rectum in prostate-containing tomography sections [15], $110 \mathrm{~mm}$ of rectum starting from anal verge $[12,16]$, or the anal verge to the rectosigmoid flexure [10,17-19].

One important drawback of using different rectal definitions and contouring methods is the resultant difficulty in interpreting the outcomes of different studies. Thus, we planned to compare four different rectal volume definition techniques and dependent irradiated percent rectal volumes on predicting rectal toxicity in patients with localized prostate cancer treated with 3DCRT, which will be a guide for evaluating the rectal toxicity wherein the rectal contouring technique used.

\section{Methods}

\section{Patient Data}

A total of 118patients with histological proof of prostate adenocarcinoma was treated with 3D-CRT between January 2007 and February 2008 in the Department of Radiation Oncology at Baskent University. We analyzed clinical and dosimetric data of 94 eligible patients. Eligibility criteria were as follows: Eastern Cooperative Oncology Group performance status (PS) of 0 to 2; age between 18 and 70 years; non-prostatectomised; no prior chemotherapy or abdominal irradiation; no distant metastasis; no contraindication for RT. Invariably, all eligible patients were treated with the same technique and the same doses, and any deviations from either the technique or dose were reasons for exclusion from the study. The clinical and dosimetric records of patients with stage T1c-T3 (American Joint Committee on Cancer, 1997 staging system) prostate cancer were used in this analysis. Prostate speci- mens were scored with the Gleason grading system. According to our current protocol, all patients were treated with 3 months of neoadjuvant total androgen blockage prior to planned irradiation. Baskent University's Institutional Review Board approved this study design.

\section{Treatment Planning}

As part of treatment planning, all patients underwent a CT scan with $2.5-\mathrm{mm}$ slice thickness. During the scan, patients were in supine with their feet fixed in a commercially available knee support device, an emptied rectum, and comfortably full bladder. Patients were asked to empty their rectum before treatment, no enema or other laxatives were used before planning CT and during treatment. The CTV was defined as the entire prostate and seminal vesicles. A $1-\mathrm{cm}$ margin was added to the CTV to define the planning target volume (PTV). The treatment volume included an additional $0.7-\mathrm{cm}$ margin for beam penumbra in all directions, except for the posterior margin, which overlaps the rectum; thus, posteriorly, a $0.5-\mathrm{cm}$ margin was added for reducing rectal toxicity. The isocenter was positioned in the center of the PTV and beams were shaped with multi-leaf collimators (MLC; Varian DHX 3323, Varian Medical Systems, Palo Alto, California, USA).

The exposed rectum was defined in four different ways for all 94 patients as depicted in Table 1. All target and organ at risk volumes were defined and contoured by the same physician. Intra-observer variability was also assessed on randomly selected 10 sample patients by a blind repetition of rectum contouring on randomly chosen CT scans. The mean intra-observer variability was $0.7 \mathrm{~mm}$ in the cranial and $0.9 \mathrm{~mm}$ in the caudal directions, respectively.

All treatments were planned with a six-field technique using a treatment planning system (Eclipse ${ }^{\circledast}$, Varian Medical Systems, Palo Alto, California, USA). A total of 70 Gy ( $2 \mathrm{~Gy} / \mathrm{fr}$, daily, Monday through Friday) was delivered using 18-MV photons. Portal images obtained from the anterior set-up and two lateral fields on the first treatment day and once weekly, or more (if necessary), during the RT period, were used to confirm field verifications by comparing them with digitally reconstructed radiographs. The portal images were reviewed by the treating physician.

\section{Table I: Rectum contouring techniques}

\begin{tabular}{ll}
\hline Methods & Techniques \\
\hline I & All prostate-containing CT sections \\
2 & I cm above and below PTV-containing sections \\
3 & I I0 mm of rectum starting from the anal verge \\
4 & Anal verge to the sigmoid flexure
\end{tabular}




\section{DVH Analysis}

The dose distribution of each plan for each patient's rectum was established and the doses were re-calculated for the different rectal volumes lengths. The DVHs created for each patient and methods were used to perform intermethod comparisons. Our analysis included the percent volume of rectum irradiated with certain dose levels (30 to $70 \mathrm{~Gy}$, in $10 \mathrm{~Gy}$ increments) evident on DVHs created for each method, and their possible predictive role on rectal toxicity incidence and severity. All doses represent total doses that have not been corrected for fractionation.

\section{Toxicity Score}

Side effects manifested within 90 days from the initiation of RT were considered "acute", and "late" those manifest thereafter. Rectal toxicities were graded according to the Radiation Therapy Oncology Group (RTOG) toxicity scores [20]. The rectal toxicity grades are: grade $1=$ minor symptoms requiring no treatment; grade 2 = symptoms that respond to simple management; grade 3 = distressing symptoms affecting lifestyle and necessitating hospital admission; grade 4 = symptoms necessitating a major surgical procedure (laparatomy, colostomy, long stay in hospital); and grade 5 = death. Grades 1 and 2 rectal bleeding is defined as incidental or intermittent bleeding requiring no treatment or responding to simple outpatient management, respectively; grade 3 rectal bleeding is defined as bleeding that requires a blood transfusion or laser cauterization.

During the RT course, all eligible patients were evaluated on the same day of the week for toxicity scoring, unless a patient required more frequent visits. In the medical records, the type of toxicity and its grade, the time of occurrence, as well as the prescribed medications and doses were systematically reported.

\section{Follow-up}

The length of follow-up was calculated from the first date of 3DCRT. According to the medical records, follow-up visits included a thorough physical examination, serum total and free prostate specific antigen (PSA), and testosterone levels, complete blood count and serum biochemistry, and pelvic MRI every 6 months. At each visit, detailed genitourinary and gastrointestinal system toxicities were assessed. The patients were first seen 6 weeks after the completion of RT and every 3 months or more frequently, if necessary, thereafter.

\section{Statistical Analysis}

The dosimetric variables considered were rectal volume, maximum and mean dose to the rectal volume (Dmax and Dmean, respectively), and volumes (percentage and absolute) of rectum receiving $30 \mathrm{~Gy}, 40 \mathrm{~Gy}, 50 \mathrm{~Gy}, 60 \mathrm{~Gy}$, and 70 Gy. For each patient and each technique, DVHs were compared for both dosimetric assessment and their predictive value on rectal toxicity. The Fisher's exact test was used to compare qualitative variables and the Student's $t$ means comparison test was used for continuous variables. The median values of these differences were compared using the Wilcoxon signed rank test to evaluate if they were significantly different from zero. A $p \leq 0.05$ (two-sided) was considered significant for all statistical tests.

\section{Results}

Total 94 of 118 eligible patients were evaluated. 26 patients were excluded from the study, because, 16 patients were treated after radical prostatectomy, 6 patients were treated with pelvic box technique because of lymph node metastasis, and 2 patients did not finish the sheduled treatment ( 1 with myocardial infarction, 1 with no reason). The patient and disease characteristics are summarized in Table 2. All 94 patients were eligible for toxicity analysis and no patient was lost to follow-up; the median follow-up interval was 13.1 months (range: 321.6 months). The treatment protocol was well tolerated in general with no report of grade 4 or 5 acute or late toxicity. Sixteen patients $(17 \%)$ completed the treatment without any significant complications. Rectal toxicities of grade 1 to 3 were reported in 34 (36\%), 36 (38\%), and 8 (9\%) patients, respectively. Rectal bleeding was reported in $13(14 \%)$ patients, and were graded as grade 2 in 12 $(13 \%)$, and grade 3 in the remaining $1(1 \%)$. This latter patient was presented at the $9^{\text {th }}$ month after 3DCRT and fared well following two courses of laser cauterization.

The median prostate and seminal vesicle volumes were 38 $\mathrm{cm}^{3}$ (range: $18-111.7 \mathrm{~cm}^{3}$ ) and $13 \mathrm{~cm}^{3}$ (range: $4.8-28.8$ $\mathrm{cm}^{3}$ ), respectively. The median prostate, seminal vesicle, and PTV doses were 69.7 Gy (range: 68.5 - 71.3 Gy), 69.8

Table 2: Patient characteristics.

\begin{tabular}{cc}
\hline Patients & \\
\hline Age (years) & \\
Median & 69 \\
Range & $48-82$ \\
Pretreatment PSA & $\mathbf{n ~ ( \% )}$ \\
$\leq 10 \mathrm{ng} / \mathrm{mL}$ & $37(39)$ \\
$>10 \mathrm{ng} / \mathrm{mL}$ & $57(6 \mathrm{l})$ \\
Gleason score & $\mathbf{n}(\%)$ \\
6 & $55(59)$ \\
7 & $30(32)$ \\
8 & $3(3)$ \\
9 & $6(6)$ \\
Stage & $\mathbf{n ~ ( \% )}$ \\
T1 & $12(13)$ \\
T2 & $63(67)$ \\
T3 & $19(20)$ \\
\hline
\end{tabular}


Gy (range: 68.4 - 72.0 Gy), and 70.0 Gy (range: 68.7 $71.5 \mathrm{~Gy})$, respectively.

Table 3 shows the median rectum volumes using the different contouring techniques. As expected, compared to methods 1 and 2, relatively larger rectum volumes were contoured in methods 3 and 4 . Table 4 shows the comparison of rectum minimum, maximum and mean doses and percentage of rectal volumes receiving different doses based on these different techniques. The mean rectal doses and percentage of rectal volumes receiving different dose levels were higher with contouring techniques that resulted in small rectal volumes (method 1) than with contouring techniques that resulted in average and large rectal volumes (method 3). Thus, for example, the mean rectum dose and V70 were higher in method 1 (57.5 Gy and $32.9 \%$, respectively) than in method 3 (49.6 Gy and $24.3 \%$, respectively).

The comparison of rectal minimum and mean doses, V30, V40, V50, V60 and V70 Gy revealed significantly higher doses for method 1 compared to the other methods; the lowest mean rectal doses and percentage of rectal volumes at different dose levels were obtained with the technique used in method 3. The minimum and mean rectal doses significantly differed in each method. Similarly, statistically significant differences were established for the percentage of rectum volumes receiving $30 \mathrm{~Gy}, 40 \mathrm{~Gy}, 50 \mathrm{~Gy}$, $60 \mathrm{~Gy}$, and $70 \mathrm{~Gy}$, respectively.

Acute rectal toxicity was closely associated with the mean rectum doses and V30 Gy, V40 Gy, V50 Gy, V60 Gy and V70 Gy points for all contouring techniques. As shown in Table 5 mean rectal doses and V70 Gy were significantly higher in patients with Grade 2 or more rectal toxicity compared to patients with or without Grade 1 rectal toxicity. The mean rectal dose in patients with Grade 2 or more rectal toxicity was lowest in method 3 (52.4 Gy) and highest in method 1(61.0 Gy). Likewise V70 Gy values were higher in methods 1 and 2 (42.3\% and 37.3\%) compared to methods 3 and 4 (32.5\% and 33.4\%), respectively.

When rectal bleeding was evaluated Wilcoxon test revealed that, in method 2 , the percentage of rectal vol-

Table 3: The Median Rectum Volumes

\begin{tabular}{cc}
\hline Methods & Volume in $\mathrm{cm}^{3}(\min -\mathrm{max})$ \\
\hline & Rectum \\
2 & $43.6(22.0-147.3)$ \\
3 & $54.7(29.8-161.4)$ \\
4 & $63.0(36.5-175.3)$ \\
& $60.5(30.5-176.2)$ \\
\hline
\end{tabular}

umes those received $\geq 70$ Gy were $30.8 \%$ and $22.5 \%$ for patients with and without rectal bleeding $(p=0.03)$, respectively. Similarly in method 3 , the percentage of rectal volume that received $\geq 70$ Gy was $26.9 \%$ and $18.1 \%$ in patients with and without bleeding $(p=0.006)$. The mean rectal dose was found to be a significant predictor of rectal bleeding only in method 3; mean rectal doses were 48.8 Gy and 44.4 Gy for patients with and without bleeding ( $p$ $=0.02$ ). No significant correlation was found for low or moderate dose levels.

\section{Discussion}

In this study, four different rectum contouring techniques were assessed, and the impact of the contouring techniques on DVH and acute rectal toxicity and rectal bleeding was evaluated. We clearly demonstrated that mean rectal dose and rectal volume receiving a high dose $(\geq 70$ $\mathrm{Gy})$ are the most important predictive factors for acute rectal toxicity and rectal bleeding, and varies according to rectal contpuring techniques. This significance was assessed in this study with different rectum contouring techniques, and especially the method 3 revealed a significant correlation.

The primary aim of 3D-CRT in prostate carcinoma is to maximize the therapeutic ratio to deliver an effective dose to the tumor while maintaining an acceptable dose to the neighboring normal tissues. In this manner, better control of the local tumor and reduction of distant metastatic rates can be achieved by escalating the dose beyond that of conventional doses without additional toxicities $[9,21,22]$. However, toxicities such as late rectal bleeding, which is one of the dose-limiting complications, may prevent escalation of the dose and therefore adversely affect treatment outcomes. The volume of the rectum included in the high dose region is the major determinant for predicting late rectal bleeding. In recent years a number of studies evaluated the relationship between rectal toxicity and rectal irradiation, and for this purpose rectal DVHs, dose wall histograms (DWHs), and dose surface histograms (DSHs) have been used. However, the definitions of the affected rectum varied widely among the researchers $[10,12-14,16-19,23]$, and no universally accepted, conclusive result has been obtained with respect to whether DVH, DSH, or DWH is the best predictor of rectal complications, including late rectal bleeding. Nor has such a result been obtained to determine which length of the contoured rectum provides the best predictor of complications. In this current study, we compared mean rectal doses and percentage of rectal volumes receiving particular doses (30-70 Gy) via DVHs in most commonly used four rectal contouring techniques to an effort to determine the best contouring technique for prediction of rectal toxicity. 
Table 4: Median Dose-Volume Histogram and Dose-Wall Histogram data for the patients treated with 3DCRT.

\begin{tabular}{llllll}
\hline & Method I & Method 2 & Method 3 & Method 4 & $p$ \\
\hline Min dose (Gy) & 8.2 & 3.2 & 1.5 & 1.9 & 0.01 \\
Mean dose (Gy) & 57.5 & 74.0 & 49.6 & 51.1 & $<.1$ \\
Max dose (Gy) & 75.1 & 75.1 & 75.1 & 75.1 & NS \\
V30 Gy (\%) & 86.7 & 84.5 & 80.6 & 82.1 & $<0.001$ \\
V40 Gy (\%) & 78.1 & 73.3 & 68.6 & 70.7 & $<0.001$ \\
V50 Gy (\%) & 70.9 & 64.2 & 55.3 & 59.8 & $<0.001$ \\
V60 Gy (\%) & 55.6 & 48.4 & 41.7 & 45.1 & $<0.001$ \\
V70 Gy (\%) & 32.9 & 27.9 & 24.3 & 26.5 & $<0.001$ \\
\hline
\end{tabular}

The use of different rectal contouring techniques with different rectal lengths and volumes yield various radiation doses, which may result in a variety of toxicity probabilities. This issue has been addressed by various authors. One of the most important predictors of acute rectal toxicity and rectal bleeding is the rectal volume receiving a high dose (60-80 Gy) [19,24,25]. Koper et al. found that the risk of rectal bleeding increased from $10 \%$ to $63 \%$ when the irradiated rectal volume increased from $25 \%$ to $100 \%$ [17]. In that study, the rectum was contoured from the anal verge proximally to the sacroiliac joint. Michalski et al., in the preliminary report of toxicity from an intergroup trial, observed that the relative risk of developing late gastrointestinal system toxicity was two-fold greater if the total rectal volume receiving radiation dose exceeded $100 \mathrm{~cm}^{3}$; the rectum was contoured as a solid organ extending from the anus to the rectosigmoid flexure [21]. In a randomized trial, Pollack et al. reported a significant increase in rectal toxicity in patients treated with 78 Gy compared to 70 Gy [16]. The DVH calculations were performed with respect to the rectal volumes within a $11-\mathrm{cm}$ cranio-caudal segment, with no specification as to whether the rectal contents were included. The authors demonstrated that the 5-year risk of grade $\geq 2$ rectal toxicity was $37 \%$ in patients with $>25 \%$ of the rectum receiving $\geq 70$ Gy compared to $13 \%$ for patients with $<25 \%$ of the rectum receiving $\geq 70 \mathrm{~Gy}$. In addition, all grade $3 \mathrm{com}$ plications occurred when V70 exceeded $30 \%$ of the rectal volume [9]. In this current study, we clearly demonstrated that all grade $\geq 2$ acute rectal toxicities were seen in patients with $>30 \%$ of the rectum receiving $\geq 70$ Gy

Table 5: Mean rectal doses and percentage of rectal volume receiving $70 \mathrm{~Gy}(\mathrm{~V} 70 \mathrm{~Gy})$ values according to acute rectal toxicity grade groups.

\begin{tabular}{cccccccc}
\hline & \multicolumn{3}{c}{ Mean Rectal Dose (Gy) } & \multicolumn{4}{c}{ V70 Gy (\%) } \\
\hline & Grade & Grade & $p$ & Grade & Grade & $p$ \\
Method I & $5-1$ & $\geq 2$ & & $0-1$ & $\geq 2$ & \\
Method 2 & 49,4 & 61,0 & $<0.001$ & 27,2 & 42,3 & $<0.001$ \\
Method 3 & 44,5 & 52,4 & $<0.001$ & 23,0 & 37,3 & $<0.001$ \\
Method 4 & 46,1 & 54,7 & $<0.001$ & 19,8 & 32,5 & $<0.001$ \\
& & & & 20,7 & 33,4 & $<0.001$ \\
\hline
\end{tabular}

regardless of contouring techniques (Table 5). Also a significant correlation was found between rectal bleeding and rectal volume receiving $\geq 70$ Gy for rectum contoured in methods 2 and 3.

The mean rectal dose is another dosimetric factor that predicts rectal morbidity. Zapatero et al. demonstrated that the mean rectal dose and V60 Gy were closely correlated with grade 2 or worse rectal bleeding in 107 patients with prostate cancer treated with 3DCRT [25]. They found that patients with rectal bleeding had a mean rectal dose of 57 Gy compared with 46 Gy for those without bleeding $(p<$ $0.0005)$. The rectum was contoured over $150 \mathrm{~mm}$, from the anus (at the level of the ischial tuberosities) to where the rectosigmoid flexure could be identified. In the current study, we found a statistically significant correlation between rectal bleeding and mean rectal doses only when the rectum was contoured over $110 \mathrm{~mm}$ starting from the anus (method 3: 48.8 Gy for patients with rectal bleeding and 44.4 Gy for patients without rectal bleeding ( $p=$ $0.02)$ ). The fact that this correlation was significant only in method 3 may be due to the fact that this technique contours larger rectal volumes than the other techniques that we used. The rectum contoured in the study of Zapatero et al. was even longer and the rectal volume larger compared to those in method 3 of our study. Thus, mean rectal doses may significantly predict rectal bleeding for techniques contouring larger rectal volumes.

In one of the first studies that evaluated the rectal contouring problem, Geinitz et al. concluded that a uniform definition of the rectal volume should be established to achieve equivalent DVH results [26]. Boehmer et al. compared two different rectal contouring techniques: one technique included the rectum bounded by two CT slices above and below the PTV; the other technique included the rectum from the anal verge to the sigmoid colon [27]. Furthermore, the posterior half of the rectum was contoured for both volumes. The first technique resulted in significantly higher minimum and mean rectal doses than did the second technique. The authors concluded that different ways of rectal contouring significantly influence calculated doses to the rectum. In another study, Liu et al. 
compared 6 different ways of contouring the rectum in 10 patients with prostate cancer treated with a four-field boxtechnique with a total dose of 70 Gy. They concluded that absolute rectal wall volume, in addition to percent rectal volume, should be used in analyzing late rectal toxicity [24].

Our study also demonstrates that the rectal DVHs vary considerably with different rectum delineation techniques. The rectum contoured in all prostate-containing CT sections (method 1) had the largest percentage of rectum receiving a specific radiation dose, since less rectum volume was contoured. Any contouring techniques that use a longer length of the rectum will result in a smaller percentage of the contoured rectum receiving the radiation dose. Thus, the technique that contoured a $110-\mathrm{mm}$ rectal segment from the anal verge (method 3 ) resulted in lower radiation doses than the techniques that contoured shorter segments and smaller rectal volumes. This is due to the fact that the absolute volume of rectum receiving a specific dose remains constant while the percentage of rectal volume receiving a specific dose becomes reduced if the total volume contoured is larger. Therefore, with different rectal length and volume contouring techniques, the differences in the configurations of the different DVHs become apparently significant.

\section{Conclusion}

In conclusion, with a relatively larger patient population, we demonstrated that percentage of rectal volumes receiving high doses ( $\geq 70 \mathrm{~Gy}$ ) and mean rectal doses which are predictors of rectal toxicity varied in different rectum contouring techniques with differing DVHs. The rectal volume exposed to high RT doses ( $\geq 70$ Gy) seems to be a crucial determinant in predicting late rectal bleeding in almost all contouring techniques. In method 3; in which rectum was contoured $110 \mathrm{~mm}$ starting from anal verge, rectum volume was found to be higher than other methods, and a significant importance of mean rectal dose and percentage of rectal volume receiving $>70$ Gy was established. Finally, we think that, there is an urgent need for a universally accepted precise definition of rectal volumes for a systematic reliable comparison of various histograms.

\section{Competing interests}

We have no personal or financial conflict of interest and have not entered into any agreement that could interfere with our access to the data on the research, or upon our ability to analyze the data independently, to prepare manuscripts, and to publish them.

\section{Authors' contributions}

All authors read and approved the final manuscript. CO prepared the design of the manuscript and made the con- touring of the target volume and organs at risk; ET and MY collected the samples; AY gave advise on the work and helped in the interpretation of the data; EE and SS made the treatment planning; $\mathrm{CO}$ wrote the paper together with ET.

\section{Acknowledgements}

This study was accepted as oral presentation at 7th Congress of Balkan Union of Oncology from 15 to 19 October 2008.

\section{References}

I. Hanks GE, Hanlon AL, Epstein B, Horwitz EM: Dose response in prostate cancer with 8-12 years' follow-up. Int J Radiat Oncol Biol Phys 2002, 54:427-435.

2. Zelefsky MJ, Yamada Y, Fuks Z, Zhang Z, Hunt M, Cahlon O, Park J, Shippy A: Long-term results of conformal radiotherapy for prostate cancer: impact of dose escalation on biochemical tumor control and distant metastases-free survival outcomes. Int J Radiat Oncol Biol Phys 2008, 71:1028-1033.

3. Nutting CM, Corbishley CM, Sanchez-Nieto B, Cosgrove VP, Webb $S$, Dearnaley DP: Potential improvements in the therapeutic ratio of prostate cancer irradiation: dose escalation of pathologically identified tumour nodules using intensity modulated radiotherapy. $\mathrm{Br} J$ Radiol 2002, 75:I5I- |6I.

4. O'Donnell HE, Finnegan K, Eliades H, Oliveros S, Plowman PN: Redefining rectal volume and DVH for analysis of rectal morbidity risk after radiotherapy for early prostate cancer. $\mathrm{Br} J$ Radiol 2008, 81:327-332.

5. Nguyen LN, Pollack A, Zagars GK: Late effects after radiotherapy for prostate cancer in a randomized dose-response study: results of a self-assessment questionnaire. Urology 1998, 5 I:991-997.

6. Zelefsky MJ, Cowen D, Fuks Z, Shike M, Burman C, Jackson A, Venkatramen ES, Leibel SA: Long term tolerance of high dose threedimensional conformal radiotherapy in patients with localized prostate carcinoma. Cancer 1999, 85:2460-2468.

7. Schultheiss TE, Lee WR, Hunt MA, Hanlon AL, Peter RS, Hanks GE: Late GI and GU complications in the treatment of prostate cancer. Int J Radiat Oncol Biol Phys 1997, 37:3-II.

8. Boersma LJ, Brink M van den, Bruce AM, Shouman T, Gras L, te Velde $A$, Lebesque JV: Estimation of the incidence of late bladder and rectum complications after high-dose (70-78 GY) conformal radiotherapy for prostate cancer, using dose-volume histograms. Int J Radiat Oncol Biol Phys 1998, 41:83-92.

9. Storey MR, Pollack A, Zagars G, Smith L, Antolak J, Rosen I: Complications from radiotherapy dose escalation in prostate cancer: preliminary results of a randomized trial. Int J Radiat Oncol Biol Phys 2000, 48:635-642.

10. Fiorino C, Cozzarini C, Vavassori V, Sanguineti G, Bianchi C, Cattaneo GM, Foppiano F, Magli A, Piazzolla A: Relationships between DVHs and late rectal bleeding after radiotherapy for prostate cancer: analysis of a large group of patients pooled from three institutions. Radiother Oncol 2002, 64: I- 12.

II. Fiorino C, Sanguineti G, Cozzarini C, Fellin G, Foppiano F, Menegotti L, Piazzolla A, Vavassori V, Valdagni R: Rectal dose-volume constraints in high-dose radiotherapy of localized prostate cancer. Int J Radiat Oncol Biol Phys 2003, 57:953-962.

12. Huang EH, Pollack A, Levy L, Starkschall G, Dong L, Rosen I, Kuban DA: Late rectal toxicity: dose-volume effects of conformal radiotherapy for prostate cancer. Int J Radiat Oncol Biol Phys 2002, 54:|3|4-|32|.

13. Beckendorf V, Guerif S, Le Prise E, Cosset JM, Lefloch O, Chauvet B, Salem N, Chapet O, Bourdin S, Bachaud JM, Maingon P, Lagrange JL, Malissard L, Simon JM, Pommier P, Hay MH, Dubray B, Luporsi E, Bey $P$ : The GETUG 70 Gy vs. 80 Gy randomized trial for localized prostate cancer: feasibility and acute toxicity. Int J Radiat Oncol Biol Phys 2004, 60:1056-1065.

14. Miralbell R, Taussky D, Rinaldi O, Lomax A, Canales S, Escude L, Nouet $P$, Ozsoy O, Rouzaud M: Influence of rectal volume changes during radiotherapy for prostate cancer: a predictive model for mild-to-moderate late rectal toxicity. Int J Radiat Oncol Biol Phys 2003, 57:I280-I 284. 
15. Chism DB, Horwitz EM, Hanlon AL, Pinover WH, Mitra RK, Hanks GE: Late morbidity profiles in prostate cancer patients treated to 79-84 Gy by a simple four-field coplanar beam arrangement. Int J Radiat Oncol Biol Phys 2003, 55:7I-77.

16. Pollack A, Zagars GK, Starkschall G, Antolak JA, Lee JJ, Huang E, von Eschenbach AC, Kuban DA, Rosen I: Prostate cancer radiation dose response: results of the M. D. Anderson phase III randomized trial. Int J Radiat Oncol Biol Phys 2002, 53:1097-I I 05.

17. Koper PC, Heemsbergen WD, Hoogeman MS, Jansen PP, Hart GA, Wijnmaalen AJ, van Os M, Boersma LJ, Lebesque JV, Levendag P: Impact of volume and location of irradiated rectum wall on rectal blood loss after radiotherapy of prostate cancer. Int J Radiat Oncol Biol Phys 2004, 58: I072-1082.

18. Peeters ST, Heemsbergen WD, van Putten WL, Slot A, Tabak $H$, Mens JW, Lebesque JV, Koper PC: Acute and late complications after radiotherapy for prostate cancer: results of a multicenter randomized trial comparing $68 \mathrm{~Gy}$ to $78 \mathrm{~Gy}$. Int J Radiat Oncol Biol Phys 2005, 61:1019-1034.

19. Nuyttens JJ, Milito S, Rust PF, Turrisi AT 3rd: Dose-volume relationship for acute side effects during high dose conformal radiotherapy for prostate cancer. Radiother Oncol 2002, 64:209-2I4.

20. Lawton CA, Won M, Pilepich MV, Asbell SO, Shipley WU, Hanks GE, Cox JD, Perez CA, Sause WT, Doggett SR: Long-term treatment sequelae following external beam irradiation for adenocarcinoma of the prostate: analysis of RTOG studies 7506 and 7706. Int J Radiat Oncol Biol Phys 1991, 21:935-939.

21. Michalski JM, Purdy JA, Winter K, Roach M 3rd, Vijayakumar S, Sandler HM, Markoe AM, Ritter MA, Russell KJ, Sailer S, Harms WB, Perez CA, Wilder RB, Hanks GE, Cox JD: Preliminary report of toxicity following 3D radiation therapy for prostate cancer on 3DOG/RTOG 9406. Int J Radiat Oncol Biol Phys 2000, 46:39I-402.

22. Pollack A, Smith LG, von Eschenbach AC: External beam radiotherapy dose response characteristics of II 27 men with prostate cancer treated in the PSA era. Int J Radiat Oncol Biol Phys 2000, 48:507-5I2.

23. Fiorino C, Vavassori V, Sanguineti G, Bianchi C, Cattaneo GM, Piazzolla $A$, Cozzarini $C$ : Rectum contouring variability in patients treated for prostate cancer: impact on rectum dose-volume histograms and normal tissue complication probability. Radiother Oncol 2002, 63:249-255.

24. Liu M, Berthelet E, Patterson K, Dick K, Kwan W: Various techniques of contouring the rectum and their impact on rectal dose-volume histograms. Med Dosim 2003, 28:189-192

25. Zapatero A, Garcia-Vicente F, Modolell I, Alcantara P, Floriano A Cruz-Conde A, Torres J], Perez-Torrubia A: Impact of mean rectal dose on late rectal bleeding after conformal radiotherapy for prostate cancer: dose-volume effect. Int J Radiat Oncol Biol Phys 2004, 59:1343-135I.

26. Geinitz H, Zimmermann FB, Narkwong L, Kneschaurek P, Wehrmann $R$, Kuzmany A, Molls M: [Prostatic carcinoma: problems in the interpretation of rectal dose-volume histograms]. Strahlenther Onkol 2000, I76: 168-172.

27. Boehmer D, Kuczer D, Badakhshi H, Stiefel S, Kuschke W, Wernecke $K D$, Budach V: Influence of organ at risk definition on rectal dose-volume histograms in patients with prostate cancer undergoing external-beam radiotherapy. Strahlenther Onkol 2006, I 82:277-282
Publish with Bio Med Central and every scientist can read your work free of charge

"BioMed Central will be the most significant development for disseminating the results of biomedical research in our lifetime. "

Sir Paul Nurse, Cancer Research UK

Your research papers will be:

- available free of charge to the entire biomedical community

- peer reviewed and published immediately upon acceptance

- cited in PubMed and archived on PubMed Central

- yours - you keep the copyright

Submit your manuscript here:

http://www.biomedcentral.com/info/publishing_adv.asp
BioMedcentral 